\title{
ANÁLISE DO DESEMPENHO DE ATLETAS NO ESPORTE DE ORIENTAÇÃO
}

Joaquim de Jesus Picardo ${ }^{1}$, Lucas Da Silva Santos ${ }^{1}$, Paulo Henrique Aleixo ${ }^{1}$, Guilherme Akio Tamura Ozaki $^{2}$, Everton Alex Carvalho Zanuto ${ }^{1}$, Thiago Alves Garcia ${ }^{3}$, Robson Chacon Castoldi ${ }^{1}$, Regina Celi Trindade Camargo ${ }^{2}$, José Carlos Silva Camargo Filho ${ }^{2}$.

${ }^{1}$ Universidade do Oeste Paulista - UNOESTE, Departamento de Educação Física, Presidente Prudente, SP. E-mail: picardo@uol.com.br.

2Universidade Estadual Paulista "Júlio Mesquita Filho" - UNESP, Departamento de Fisioterapia, Presidente Prudente, SP.

${ }^{3}$ Universidade Estadual de Campinas - UNICAMP, Departamento de Ortopedia e Traumatologia, Campinas, SP.

\section{RESUMO}

Esta pesquisa teve o objetivo verificar o desempenho de atletas do esporte de orientação. A amostra estudada foi composta por dez atletas praticantes da modalidade, participantes do Campeonato Paulista de Orientação (CAMPOR/2016), com faixa etária entre 21 a 30 anos, pertencentes à classe de competidores "Categoria H21A" (homens acima de 21 anos de idade), com percurso com grau de dificuldade difícil e intermediário. Foi utilizado o software "SPORTident" para a obtenção dos resultados do desempenho dos atletas durante a competição. Foi verificado que os atletas observados obtiveram classificação de primeiro (1ㅇ) ao trigésimo quinto (35) lugares. Observa-se ainda que em determinados pontos foi verificada maiores diferenças, fato que influenciou na velocidade média dos atletas, sendo esta estabelecida em 1,74 $\mathrm{m} / \mathrm{s}$ no 1 o colocado e 1,24 no 35 o. Conclui-se que mesmo pertencentes à mesma categoria e com faixa etária semelhante, os atletas avaliados apresentaram desempenhos diferenciados.

Palavras-chave: Educação Física e Treinamento; Cognição, Fadiga.

\section{ANALYZE TO PERFORMANCE OF ATHLETES IN ORIENTATION SPORTS}

\begin{abstract}
The aim of study was verify the performance of athletes of the orientation sports. The sample was composed for ten athletes practices of modality, participants to Paulista Orientation Championship (CAMPOR/2016), with age to 21 and 30 years, from to competitor class "Category H21A" (mans over 21 years old), with road hard and intermediaries difficulty. Was utilized the "SPORTident" for attainment to results to performance to athletes during the competition. Was verify that athletes obtained the $1^{\text {st }}$ and $35^{\text {th }}$ places. Was showed that in some ware was verify more differences, fact that influenced in athltes, with velocity in $1,74 \mathrm{~m} / \mathrm{s}$ for $1^{\text {st }}$ and 1,24 for $35^{\text {th }}$. It is concluded that though from same category and has similar ages, the athletes evaluated showed differentiated results.
\end{abstract}

Key-Words: Physical Education and Training; Cognition, Fatigue. 


\section{INTRODUÇÃO}

A orientação pedestre é um esporte que impõe demandas consideráveis sobre as capacidades cardiovasculares e metabólicas ao praticante, podendo manifestar alto nível de condição física necessária para os atletas de elite. ${ }^{1}$ Nesse sentido os atletas de orientação pedestre apresentam alta capacidade de resistência, sendo capazes de trabalhar a uma porcentagem perto do VO2Max durante um tempo prolongado ${ }^{1}$.

O conhecimento da fisiologia específica do esporte é um elemento fundamental para a elaboração de um perfil dos desportistas, possibilitando avaliar sua condição física e obter dados para a prescrição individualizada de treinamento ${ }^{2}$. O esporte de "orientação" possui características peculiares que além do componente físico, explora e desenvolve qualidades cognitivas: o raciocínio lógico-matemático, leitura e interpretação de mapas, tomada de decisão e concentração.

A conscientização e respeito ambiental também estão presentes e proporcionam aos praticantes uma plena harmonia com a natureza. O componente lúdico é outro benefício, propiciando bem-estar, satisfação, realização pessoal e melhorando a autoestima dos seus praticantes. A Corrida de Orientação é um esporte moderno que segue princípios pedagógicos em todas suas fases: na iniciação, no aprimoramento, no aperfeiçoamento, no alto rendimento e na manutenção. Além disso, aplica e segue princípios pedagógicos, norteadores e característicos desta modalidade, justificando sua contribuição para o desenvolvimento cognitivo na Educação Física ${ }^{3}$.

A Confederação Brasileira de Orientação (CBO), entidade máxima da administração do Esporte de Orientação no país, reconhecendo sua importância e contribuição para o desenvolvimento da Educação Física Escolar como ferramenta interdisciplinar que permite a integração da Educação Física com outras disciplinas afins, elaborou o Projeto "Escola Natureza", objetivando sua inserção nos currículos escolares, em todos os níveis, como atividade capaz de auxiliar na formação integral de crianças, jovens e adultos, numa perspectiva de educação pedagógica formativa e continuada ${ }^{3}$.

Este esporte possui quatro vertentes: competitiva, ambiental, pedagógica e turística. ${ }^{3} \mathrm{~A}$ vertente competitiva constitui-se num conjunto de ações destinadas a formação do atleta, oportunizar esta modalidade a um número cada vez mais crescente de participantes pelo trabalho metódico, pedagógico e programático dos clubes, com o principal escopo de determinar o crescimento da Orientação.

Já a vertente pedagógica do "esporte de orientação" corresponde a um conjunto de ações que visam colocar o desporto a serviço da sociedade, na busca de uma prática esportiva saudável com uma melhor qualidade de ensino na preparação física e técnica, dentro de padrões metodológicos, buscando a participação e formação do sujeito para o exercício da cidadania e para a prática deste esporte como uma atividade lúdica.

Essas múltiplas possibilidades estão contidas e são bem características da corrida de "orientação", pois através do aprendizado sistematizado e metodológico das disciplinas correlatas, possibilita e oportuniza aos seus praticantes a assimilação de conhecimentos de forma natural, crescente e permanente. Observa-se uma lacuna existe nesta modalidade esportiva, que embora tenha completado 100 anos de existência em 1998, no Brasil, está sendo difundida e praticada há aproximadamente 40 anos, conta com poucos estudos científicos existentes para desenvolver eficientemente os programas de treinamento e preparação técnica e física de atletas ${ }^{3}$.

Dessa maneira, esta pesquisa teve o objetivo verificar o desempenho de atletas do esporte de orientação buscando o desenvolvimento de práticas adequadas na preparação física e técnica para atletas de alto rendimento dentro da área da Educação Física. 


\section{METODOLOGIA}

\section{Amostra}

A amostra a ser estudada foi composta por 10 atletas, praticantes da modalidade, e participantes do Campeonato Paulista de Orientação - 2016 (CAMPOR/2016), em uma das Etapas do CAMPOR/16, com faixa etária entre 21 a 30 anos, divididos em duas classes de competidores: Categoria H21A (homens acima de 21 anos de idade, com percurso com grau de dificuldade difícil) e Intermediário (realiza preparação física 2 a 3 vezes por semana, ativo há pelo menos 12 meses). Este estudo foi aprovado pelo comitê de ética local (CAAE: 48657715.7.0000.5515).

\section{Monitoramento das variáveis de deslocamento e velocidade}

O percurso foi monitorado pelo Sistema de Posicionamento Global (GPS). Assim foi possível identificar a distância percorrida e tempo gasto durante a prova. A partir da obtenção destas variáveis foi possível calcular a velocidade de cada um dos avaliados durante o percurso a partir da seguinte fórmula: Velocidade $=[\Delta$ espaço/ $/ \Delta$ tempo $]$.

Foi utilizado o software "SPORTident" de fabricação alemã para a obtenção dos resultados do desempenho dos atletas na competição na qual foi feita a coleta de dados.

\section{Este software consiste basicamente dos seguintes componentes:}

- base eletrônica montada em todos os pontos de controles do percurso da corrida de orientação tem por finalidade registrar a passagem (tempo real: hora, minuto e segundo) do atleta na base determina (ponto de controle);

- SI Card (chip eletrônico) conduzido pelo atleta durante a realização do seu percurso da corrida de orientação, tem por finalidade registrar a passagem (tempo real: hora, minuto e segundo) do atleta na base determina (ponto de controle);

- base leitora de dados, instalada após a linha de chegada tem a finalidade de verificar e confirmar ou não a passagem do atleta por todos os pontos de controles do seu percurso;

- impressora, instalada após a linha de chegada tem a finalidade apresentar o relatório final do desempenho do atleta no seu percurso.

\section{Planilha de resultados}

A planilha de resultados que o software apresenta do desempenho do atleta apresenta os seguintes dados:

a) tempo total do percurso;

b) velocidade média total (tempo/distância):

c) tempos parciais (tempo decorrido entre os pontos de controles, na sequência crescente dos pontos de controles);

d) velocidade parciais (tempo/distância) decorrido entre os pontos de controles, na sequência crescente dos pontos de controles).

\section{Interpretação dos resultados}

Os resultados foram apurados pelo sistema "Helga", que permite a obtenção dos dados necessários e indispensáveis para realizar comparação de desempenho global e parcial entre os atletas.

\section{Análise e interpretação dos dados}

Após a obtenção dos dados, foram verificados os valores de tempo total gasto durante a prova, tempo somado a partir de cada posto de coleta, velocidade média total e velocidade média em cada parte do percurso. Estabeleceu-se o critério de análise dos atletas selecionados a partir 
da colocação na prova. Desse modo, foram analisados 10 atletas, distribuídos entre a primeira (1a) e trigésima quinta colocação (35ạ).

\section{RESULTADOS}

Foi verificado que os atletas observados obtiveram classificação de primeiro (1ㅇ) ao trigésimo quinto (35) lugares (Gráfico 1).

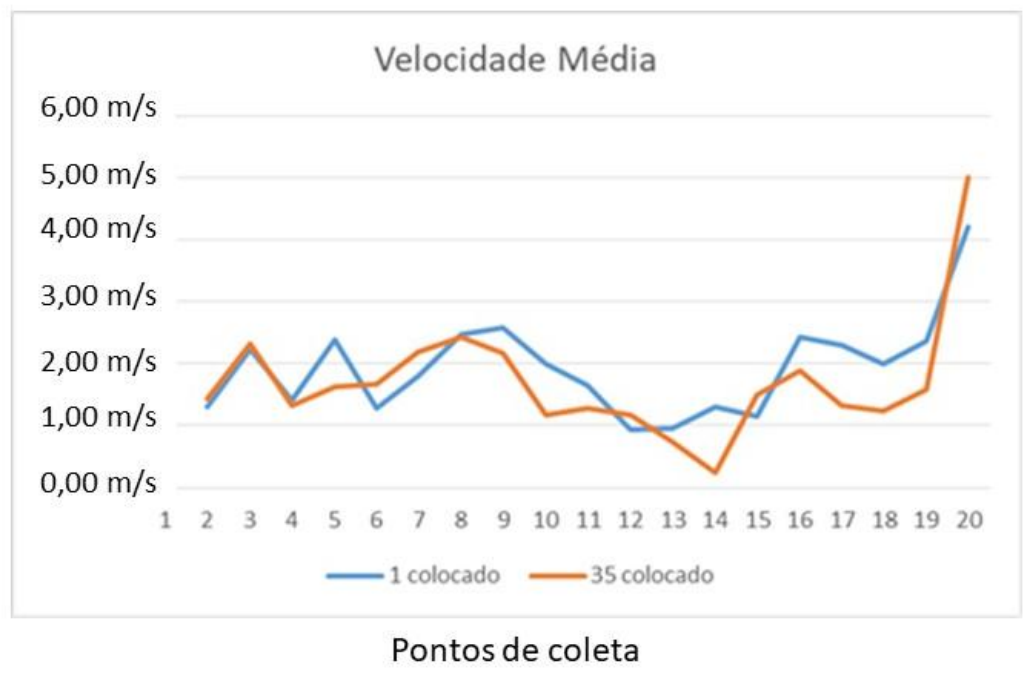

Gráfico 1. Ilustra a diferença entre atletas durante os diferentes momentos da prova.

Além disso, o primeiro colocado atingiu o final da prova com o tempo de 0:51'42" enquanto o último atleta analisado obteve o tempo de 1:12'27". Observa-se ainda que em determinados pontos foi verificada maiores diferenças, fato que influenciou na velocidade média dos atletas, sendo esta estabelecida em 1,74 m/s no 10 colocado e 1,24 no 35ㅇ (Quadro 1).

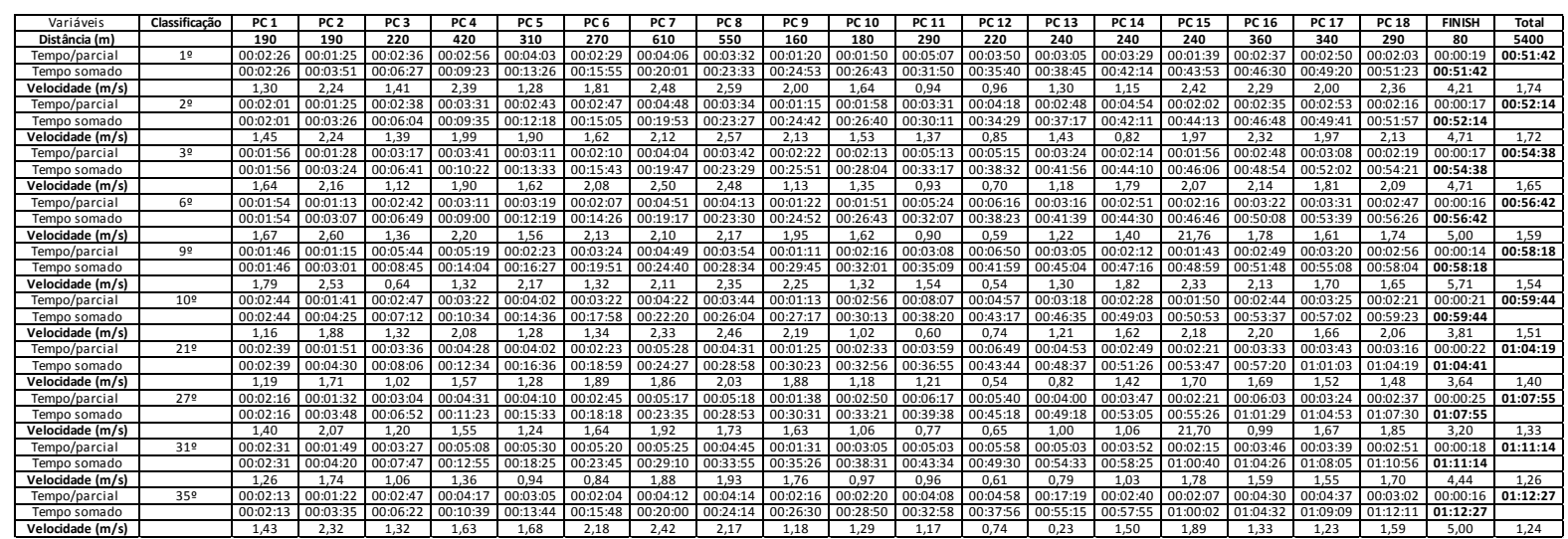

Quadro 1. Demostra o desempenho durante a prova a partir da classificação dos competidores.

\section{DISCUSSÃO}

Esta pesquisa teve o objetivo verificar o desempenho de atletas do esporte de orientação buscando o desenvolvimento de práticas adequadas na preparação física e técnica para atletas de alto rendimento dentro da área da Educação Física. Foi verificado que os atletas observados obtiveram classificação de primeiro (1으) ao trigésimo quinto (35으) lugares.

Além disso, o primeiro colocado atingiu o final da prova com o tempo de 0:51'42" enquanto o último atleta analisado obteve o tempo de 1:12'27". Observa-se ainda que em determinados pontos foi verificada maiores diferenças, fato que influenciou na velocidade média dos atletas, sendo esta estabelecida em $1,74 \mathrm{~m} / \mathrm{s}$ no 1 을 colocado e 1,24 no 35․ 
Os achados no presente estudo podem trazer informações substancias para o desenvolvimento da prática do esporte de orientação. Nota-se que apesar da proximidade dos resultados verificados por cada um dos atletas analisados, existem alguns detalhes que contribuem para a melhor colocação ao final da prova.

Dentre diversos fatores que podem contribuir para o resultado final, a tomada de decisão é certamente um dos mais determinantes. Além disso, verifica-se que a falta de preparação física pode contribuir para um desfecho negativo ao final de cada percurso. Existem relatos na literatura de que a fadiga compromete o desempenho intelectual do atleta e nesse caso, pode fazer com o desempenho físico possa ser comprometido de forma conjunta ${ }^{4}$.

A Orientação Pedestre pode ser definida como uma corrida individual, contrarrelógio, em terreno desconhecido e variado, geralmente de floresta ou montanha, utilizando um mapa e, eventualmente, uma bússola ${ }^{5}$. Apesar de relativamente recente, a corrida de orientação pode ser encarada como uma modalidade esportiva crescente nos dias atuais, na qual gera a possibilidade de contribuir para o desenvolvimento de amplos aspectos do ser humano, seja ele físico ou mental.

Nesse contexto, alguns autores destacam a importância desta modalidade no desenvolvimento da capacidade física de forma global. Na qual o esporte passe por uma transformação didático-pedagógica e que o "campo de diferentes e significativas possibilidades de um se-movimentar", ou seja, correr, saltar e arremessar/lançar ${ }^{6}$.

No entanto, observa-se uma lacuna existe nesta modalidade esportiva, que embora tenha completado 100 anos de existência em 1998, no Brasil, está sendo difundida e praticada há aproximadamente 40 anos, conta com poucos estudos científicos existentes para desenvolver eficientemente os programas de treinamento e preparação técnica e física de atletas. Sendo assim, a contribuição deste estudo destina-se ao desenvolvimento do esporte "orientação", de forma a melhorar a performance de atletas de alto rendimento.

Há uma necessidade latente de estudos científicos voltados para formação, aperfeiçoamento e manutenção do treinamento adequado, visando uma preparação física e técnica centradas principalmente na influência da fadiga no seu rendimento global. Estudos futuros que busquem investigar o comportamento das variáveis fisiológicas durante a prática desta modalidade podem vir a contribuir com os resultados apresentados na presente investigação.

\section{CONCLUSÃO}

Conclui-se que mesmo pertencentes à mesma categoria e com faixa etária semelhante, os atletas apresentaram desempenhos diferenciados, sendo classificados de 10 ao 35ㅇ lugar. Foi possível observar ainda, que em determinados pontos, foi verificada maiores diferenças, fato que influenciou na velocidade média dos atletas, sendo esta estabelecida em 1,74 m/s e 1,24, respectivamente.

\section{REFERÊNCIAS}

1. Da Silva SRD, Fraga CHW, Gonçalves M. Efeito da fadiga muscular na biomecânica da corrida: uma revisão. Motriz. Revista de Educação Física. UNESP, 2007;13(3), 225-235.

2. Fernández LS, Ruíz MR. Valoración de la condición aeróbica del corredor de orientación a pie de alto nivel español. Archivos de medicina del deporte: revista de la Federación Española de Medicina del Deporte y de la Confederación Iberoamericana de Medicina del Deporte. 2013; (158), 359-364. 
3. CBO - CONFEDERAÇÃO BRASILEIRA DE ORIENTAÇÃO. Projeto Escola Natureza. 2001.Disponível em http://www.cbo.org.br/projetos. Acesso em 15 Mar 2015.

4. Powers S K, Howley ET, Ikeda M, Navarro F, Bacurau RFP. Fisiologia do exercício: teoria e aplicação ao condicionamento e ao desempenho. 2000.

5. Aires A, Quinta-Nova LC, Santos L, Pires N, Costa R, Ferreira R. Orientação desporto com pés e cabeça. 2. ed. Portugal;2011 Disponível em http://www.fpo.pt Acesso em 15 Mar 2015.

6. Kunz E. Transformação didático-pedagógica do esporte. Ed. UNIJUI, 2000. p. 26. 